\title{
Impatto del mese di nascita sullo sviluppo di malattie autoimmuni della tiroide nel Regno Unito e in Europa
}

\author{
Michele Marinò
}

Pubblicato online: 20 settembre 2014

(C) Springer International Publishing AG 2014

Commento a:

Impact of month of birth on the development of autoimmune thyroid disease in the UK and Europe. A. Hamilton, P.R. Newby, J.D. Carr-Smith, G. Disanto, A. Allahabadia, M. Armitage, T.H. Brix, K. Chatterjee, J.M. Connell, L. Hegedus, P.J. Hunt, J.H. Lazarus, S.H. Pearce, B.G. Robinson, J.C. Taylor, B. Vaidya, J.A. Wass, W.M. Wiersinga, A.P. Weetman, S. Ramagopalan, J.A. Franklyn, S.C. Gough, M.J. Simmonds.

J Clin Endocrinol Metab (2014) doi:10.1210/jc.20141270

Una della ipotesi patogenetiche per le malattie autoimmuni, comprese quelle della tiroide, prevede che infezioni batteriche o virali possano determinarne l'insorgenza in soggetti predisposti. La somiglianza tra proteine virali o batteriche e autoantigeni (il cosiddetto molecular mimicry) sarebbe alla base di questo fenomeno. Sulla stessa linea è la cosiddetta hygiene hypothesis of autoimmunity, peraltro mai dimostrata con certezza, che prevede che le malattie autoimmuni sarebbero in qualche modo facilitate da condizioni igieniche buone, ovvero dalla minore esposizione sistematica ad agenti infettivi, e di conseguenza dalla maggiore suscettibilità a infezioni sporadiche.

In accordo con l'ipotesi di un ruolo delle infezioni nella patogenesi delle malattie autoimmuni della tiroide, in passa- to è stata osservata una differenza nell'incidenza delle stesse nei vari periodi dell'anno, con un aumento nei mesi autunnali e invernali, quelli con maggior frequenza di infezioni virali o batteriche.

Nel presente studio veniva valutata l'epoca della diagnosi delle malattie autoimmuni della tiroide in tre ampie casistiche controllate, con lo scopo di verificarne l'eventuale stagionalità. I pazienti studiati provenivano dal National UK Caucasian AITD Case Control Collection (2746 con morbo di Basedow e 502 con tiroidite autoimmune, confrontati con 1.423.716 controlli), dal National UK Caucasian GD Family Collection (239 con morbo di Basedow confrontati con 227 controlli) e dall'OXAGEN AITD Caucasian Family Collection ( 885 con morbo di Basedow, 717 con tiroidite autoimmune e 794 controlli).

Complessivamente i risultati escludevano un'associazione tra mese o stagione di nascita e insorgenza di malattie autoimmuni della tiroide, contrariamente a quanto osservato in passato. Benché tale risultato sia sostanzialmente negativo, e peraltro ottenuto in casistiche sufficientemente ampie per potersi considerare conclusivo, non può ritenersi in ogni caso sufficiente ad escludere con certezza un ruolo delle infezioni batteriche o virali nella patogenesi delle malattie autoimmuni tiroidee, che potrebbe essere del tutto indipendente dalla stagionalità.

\footnotetext{
M. Marinò (凶)

U.O. Endocrinologia 1, Università di Pisa, Pisa, Italia

e-mail: Michele.marino@med.unipi.it
} 